\title{
Relación entre carga parasitaria y linfocitos Th1 en biopsias de piel de perros con leishmaniosis
}

\author{
Catuogno, M.S.; Burna, A.N.; A.Brunel, F.; Díaz, B.; S.Negrette, M. \\ Cát. Patol. Gral., Fac. Cs. Veterinarias, Univ. Nac. Nord. UNNE, \\ Corrientes, Argentina. E-mail: mscatuogno@gmail.com
}

\begin{abstract}
Resumen
Catuogno, M.S.; Burna, A.N.; A.Brunel, F.; Díaz, B.; S.Negrette, M.: Relación entre carga parasitaria y linfocitos Th1 en biopsias de piel de perros con leishmaniosis. Rev. Vet. 31: 1, 3-7, 2020. La leishmaniosis visceral (LV) es una enfermedad sistémica crónica severa causada por Leishmania donovani o L. infantum. Varios estudios han demostrado que la susceptibilidad a LV está relacionada a altos títulos de anticuerpos circulantes y a una depresión de la inmunidad mediada por linfocitos Th1, principalmente con disminución de la producción de interferon (IFN- $\gamma$ ) e interleukina (IL-12). Similar a la humana, la LV canina está caracterizada por activación policlonal de células B e inmunosupresión específica. El objetivo del trabajo fue establecer la relación entre la carga parasitaria y la respuesta inmune en perros con LV. Se seleccionaron pacientes caninos con diagnóstico serológico reactivo a LV y se les extrajeron muestras para examen parasitológico directo y biopsias de piel del pabellón auricular para efectuar la técnica inmunohistoquímica con el fin de detectar linfocitos Th1 en relación con la carga parasitaria. En muestras de animales sin síntomas que registraban una elevada carga parasitaria, la cantidad de Th1 no superaba las 4 células por campo de mayor aumento, mientras que, en las muestras donde prevalecían cargas moderadas y bajas de parásitos, la cantidad de Th1 superaban las 8 células por campo de mayor aumento, pudiendo llegar hasta 10. De las muestras provenientes de animales con síntomas se observó que en aquéllas con moderada o elevada carga de parásitos, la cantidad de linfocitos Th1 no superaba las 5 células, mientras que las muestras con baja carga parasitaria superaban las 8 células por campo de mayor aumento. Se concluye que la presencia o ausencia de sintomatología en caninos con LV no depende de la carga parasitaria encontrada en las biopsias de piel. Por otra parte, se observó que la alta carga parasitaria se relaciona con inmunosupresión mediada por linfocitos Th1, mientras que en los animales con alta respuesta inmunológica, la carga parasitaria fue baja.
\end{abstract}

Palabras clave: perros, leishmaniosis, linfocitos Th1, inmunohistoquímica, biopsia de piel, carga parasitaria.

\begin{abstract}
Catuogno, M.S.; Burna, A.N.; A.Brunel, F.; Díaz, B.; S.Negrette, M.: Relationship between parasitic load and Th1 lymphocytes in skin's biopsies of dogs with leishmaniasis. Rev. Vet 31: 1, 3-7, 2020. Visceral leishmaniasis (VL) is a severe chronic systemic disease caused by Leishmania donovani or L. infantum. Several studies have shown that susceptibility to VL is related to high levels of circulating antibodies and a depression of the immunity mediated by Th1 cells, mainly with decreased production of interferon (IFN- $\gamma$ ) and interleukin-12 (IL-12). Like human leishmaniasis, active canine VL is characterized by polyclonal activation of B-cells and specific immune suppression. Canine patients with reactive serological diagnosis to Leishmania sp were selected and samples were taken for direct parasitological examination and skin biopsies of the pinna for the immune-histochemistry technique, in order to detect Th1 lymphocytes in relation to parasitic amount. In samples of animals without symptoms and high parasite load, the number of Th1 lymphocytes did not exceed 4 cells per field, while in the samples with moderate and low parasite loads, the number of Th1 lymphocytes exceeded 8 cells per field of higher magnification, reaching up to 10. In animals with symptoms, it was observed that in samples with moderate and high parasite loads, the number of Thl lymphocytes did not exceed 5 cells, while the samples with low parasitic amount exceeded 8 cells per field of higher magnification. It is concluded that the presence or absence of symptoms in canines with VL does not depend on the parasitic amount found in skin biopsies. On the other hand, it was observed that the high parasitic amount is related
\end{abstract}


to immune suppression mediated by Th1 lymphocytes, while in animals with high immunological response, the number of parasites is low.

Key words: dogs, leishmaniasis, lymphocytes Th1, immunohistochemistry, skin biopsy, parasitic amount.

\section{INTRODUCCIÓN}

La leishmaniosis visceral (LV) es una enfermedad sistémica crónica severa, causada por Leishmania donovani o L. infantum ${ }^{23}$. La afección se transmite a huéspedes humanos y animales a través de la picadura de moscas hembras del género Phlebotomus en el "viejo mundo" y Lutzomya en el "nuevo mundo" 11.

La LV está caracterizada clínicamente por fiebre prolongada, debilidad, anorexia, pérdida de peso, hepatomegalia, esplenomegalia, hipergammaglobulinemia y pancitopenia. Sin un tratamiento, la enfermedad puede progresar y culminar en una severa caquexia, enfermedad multisistémica, sangrado, infecciones secundarias y muerte ${ }^{10,16}$.

En la LV los componentes de la saliva del vector ejercen en el hospedador un efecto vasodilatador, anticoagulante, inmunomodulador y quimioatrayente de células inmunitarias. Esto induce un reclutamiento hacia el sitio de infección de células polimorfonucleares, macrófagos y posteriormente células natural killer (NK) y células dendríticas (CD).

La respuesta inmunitaria innata se inicia después de que estas células inmunitarias equipadas con receptores, hayan contactado con leishmanias y reconocido sus patrones moleculares patogénicos, así como otras moléculas de leishmanias conservadas. Las primeras células reclutadas del sitio de infección por protozoarios son los polimorfonu-

nucleares, en concreto los granulocitos neutrófilos, cuya función principal implica la fagocitosis y la eliminación de patógenos en respuestas inmunitarias innatas ${ }^{12}$.

Diversos estudios han descrito la presencia de parásitos de leishmanias en las $\mathrm{CD}$ de huéspedes infectados. Las CD cumplen un papel esencial como células presentadoras de antígenos, siendo un nexo entre las respuestas inmunitarias innatas y las adquiridas. Las células precursoras de $\mathrm{CD}$ se producen en la médula ósea y circulan hacia los tejidos donde residen como células inmaduras con una alta capacidad fagocítica ${ }^{6}$.

Durante el contacto con el parásito, las $\mathrm{CD}$ inmaduras interaccionan a través de sus receptores con diversos antígenos de Leishmania $s p$. Después de la interacción entre los parásitos y las $\mathrm{CD}$, estas últimas sufren un proceso de maduración durante el cual aumentan la expresión en superficie de $\mathrm{CMH}$ (complejo mayor de histocompatibilidad) de tipo I y II, así como de moléculas co-estimuladoras. Las CD infectadas son capaces de sintetizar interleukinas (IL-12, a diferencia de los macrófagos), que conducen a la generación de un perfil de tipo Th1 ${ }^{24}$.
Dependiendo del tipo de sinapsis que se establezcan, se promueven unos u otros fenotipos Leishmania-específicos de tipo Th1/Th2 (TCD4+), citotóxico (TCD8+) o supresor (Treg) ${ }^{4}$. Durante la década de 1980 se profundizó el conocimiento de la funcionalidad de los linfocitos $\mathrm{T}$ y se identificaron dos tipos de respuestas colaboradoras: la Th1 (inmunidad celular o retardada) y la Th2 (inmunidad humoral). Las Th1 son altamente efectivas en la eliminación de patógenos intracelulares y las Th2 son de gran importancia en la eliminación de microorganismos extracelulares y parásitos ${ }^{1}$.

Los granulocitos pueden liberar citoquinas proinflamatorias que atraen CD (células dendríticas) y su interacción a través de un receptor específico orquesta respuestas inmunitarias de tipo Th1 ${ }^{22}$. Estas dos respuestas, fundamentalmente de tipo celular, dirigidas por los linfocitos $\mathrm{T}$ helper, representan dos formas evolutivas: la Th1 se asocia a un aumento de linfoproliferación específica con niveles de inmunoglobulinas moderados o bajos y la Th2 con una linfoproliferación específica disminuida, inmunoglobulinas muy elevadas y una evolución clínica desfavorable.

En la leishmaniosis, la expansión de las células Th1 se asocia a la inmunidad protectora y la de Th2 a la progresión o persistencia de la enfermedad ${ }^{2,3,9,13}$. Se ha evidenciado que las células Th1 producen interferón (IFN- $\gamma$ ), interleukina (IL-2) y factor de necrosis tumoral (TNF- $\alpha$ ). En cuanto al papel de la IL-12 se ha sugerido que la expresión simultánea de ésta junto con IL- 2 e INF- $\gamma$ conducen a un proceso de retraso en el establecimiento de la enfermedad en perros infectados experimentalmente ${ }^{21}$.

En ese contexto, varios estudios han demostrado que la susceptibilidad a la LV está relacionada a altos títulos de anticuerpos circulantes y a una depresión de la inmunidad mediada por células $\mathrm{T}$ de tipo 1 , principalmente con disminución de la producción de IFN- $\gamma$ e interleukina (IL-12), incluyendo un marcado aumento de las interleukinas IL-4 e IL-10 17,21.

Similar a la LV humana, la LV canina activa está caracterizada por activación policlonal de células B, inmunosupresión específica y la aparición de síntomas clínicos dependiendo de la cantidad de parásitos en diferentes órganos viscerales ${ }^{19,20}$. Parece existir una interacción entre citoquinas de Th1 y Th2 durante la infección por Leishmania, y esto sugiere roles importantes para las diferentes citoquinas en la protección y patogénesis de la enfermedad ${ }^{5}$.

La respuesta inmune innata ordena el reclutamiento celular y ayuda a desarrollar el microambiente de citoquinas para el cual están preparadas las células $\mathrm{T}^{14,18}$. El control de la infección de LV depende de una exitosa 
respuesta inmune mediada por células ${ }^{15}$ en la cual el INF- $\gamma$ producido principalmente por células $\mathrm{T}$ CD4+ y células NK estimuladas por IL-12, lleva a la acción microbicida mediada por óxido nítrico ${ }^{7,8}$.

El objetivo del presente trabajo fue establecer la relación entre la carga parasitaria y la respuesta inmune en perros infestados naturalmente con Leishmania sp.

\section{MATERIAL Y MÉTODOS}

Se trabajó con un total de 20 animales, todos reaccionantes a la inmuno-cromatografía directa mediante Kala Azar Detect canino, de los cuales algunos presentaban síntomas y otros fueron asintomáticos.

Se obtuvieron muestras de esos 20 caninos, que procedían principalmente de clínicas privadas, así como también de aquellos perros que se encontraron en lugares donde se constató la presencia de flebótomos infectados, correspondientes a distintas zonas del nordeste argentino.

Se seleccionaron pacientes caninos con diagnóstico serológico reactivo a leishmania mediante prueba serológica con tiras inmunocromatográficas rk39 Kala Azar Detect canino. A esos pacientes se les extrajeron muestras para exámenes parasitológicos directos, por punción de médula ósea, extensión en portaobjetos y coloración con Giemsa.

También se obtuvieron muestras por biopsia de piel del pabellón auricular. En todos los casos se anotaron los síntomas. Con las muestras de pabellón auricular se realizó la técnica inmuno-histoquímica para detectar la proporción de linfocitos Thl en relación con la carga parasitaria. Para la obtención del anticuerpo primario se envió una muestra conservada en nitrógeno líquido de nódulo linfático de un canino naturalmente infectado con Leishmania sp al Instituto de Salud y Ambiente del Litoral (ISAL, Universidad Nacional del Litoral, Facultad de Bioquímica y Cs. Biológicas, Santa Fe).

A partir de esa muestra se elaboró el anticuerpo primario policlonal purificado para la detección de los linfocitos Th1. Se practicó la técnica inmuno-histoquímica con el desparafinado e hidratación de las muestras, inhibición de peroxidasa endógena, incubación con el anticuerpo primario y luego con el secundario. Posteriormente se recurrió al revelado mediante la aplicación de extravidina (Sigma) 1/200 durante 30 minutos en cámara húmeda, a temperatura ambiente, aplicando luego el cromógeno.

Para finalizar se contrastó con hematoxilina durante un minuto y se procedió a deshidratar y montar las muestras con bálsamo de Canadá natural. También se practicó inmuno-histoquímica para la detección de la cantidad de leishmanias, de la misma manera descripta anteriormente pero cambiando el anticuerpo primario y secundario.
Para poder relacionar la carga parasitaria con la proporción de linfocitos Th1 se evaluaron 2 muestras seriadas consecutivas de cada biopsia (una muestra coloreada para Leishmania $s p$ y otra para linfocitos Th1). La carga parasitaria se determinó como baja, moderada y elevada, así como también para la población de linfocitos Th1 (Diagrama 1).

\section{RESULTADOS}

Sobre un total de 20 animales analizados, $12(60 \%)$ presentaron síntomas y $8(40 \%)$ no los presentaron. De los animales sin sintomatología, 4 (50\%) revelaron una elevada carga parasitaria en las biopsias de pabellón auricular, 2 (25\%) carga moderada y 2 (25\%) carga baja con escasos parásitos identificables (Figura 1).

En cuanto a la proporción de linfocitos Th1 se pudo observar que en las muestras donde había una elevada carga parasitaria, la cantidad de Th1 no superaba las 3 células por campo de mayor aumento, mientras que en las muestras donde prevalecían carga moderada y baja de parásitos, la cantidad de Th1 superaba las 6 células por campo de mayor aumento (Figura 2), pudiendo llegar hasta 10.

De los animales con sintomatología, 2 (16\%) presentaron baja carga parasitaria, $2(16 \%)$ carga modera-

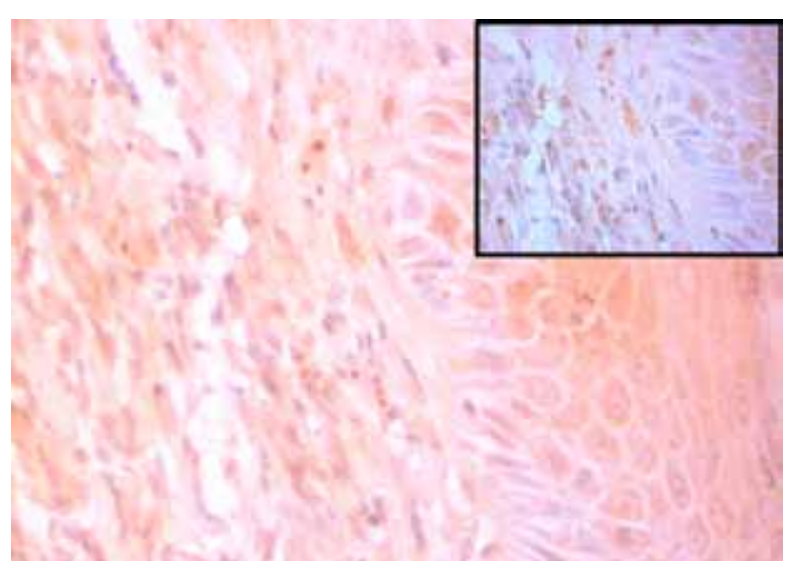

Figura 1. Piel de pabellón auricular de canino asintomático, con escasa cantidad de amastigotes de Leishmania sp (puntillado de color marrón oscuro, IHQ, $40 \mathrm{X})$. Recuadro superior con detalle a mayor aumento (IHQ, 100X). 
da y $8(67 \%)$ carga elevada de parásitos en las biopsias de piel (Figura 3).

De las muestras provenientes de animales con síntomas se observó que donde había moderada y elevada carga de parásitos, la cantidad de linfocitos Th1 no superaban las 5 células (Figura 4), mientras que las mues-

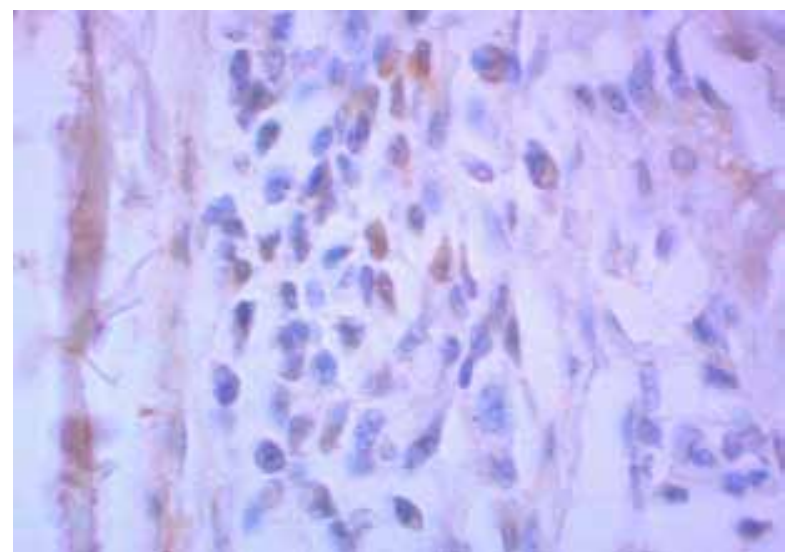

Figura 2. Misma biopsia de Figura 1, resaltando mediante inmunomarcación la presencia de 8 células Th1 en color marrón (IHQ 100X).

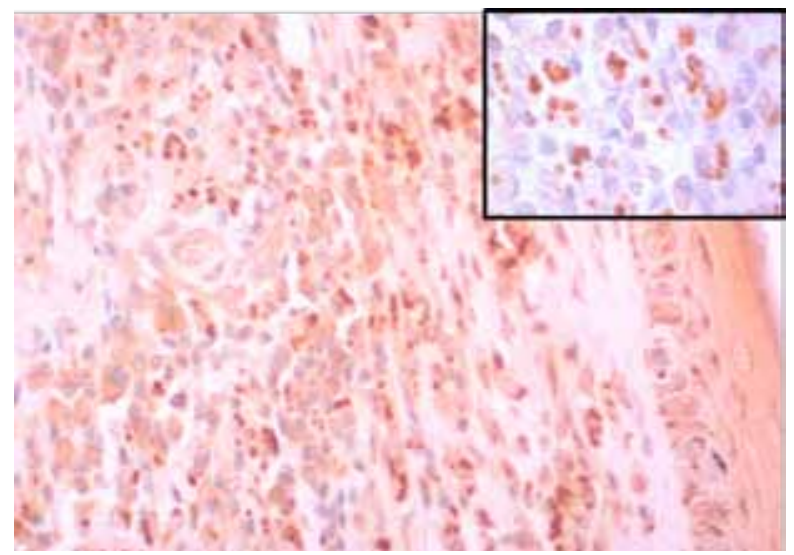

Figura 3. Biopsia de piel de pabellón auricular de canino con síntomas. Se observan abundantes amastigotes de Leishmania sp (IHQ, 40X). Recuadro: ídem a mayor aumento (IHQ, 100X).

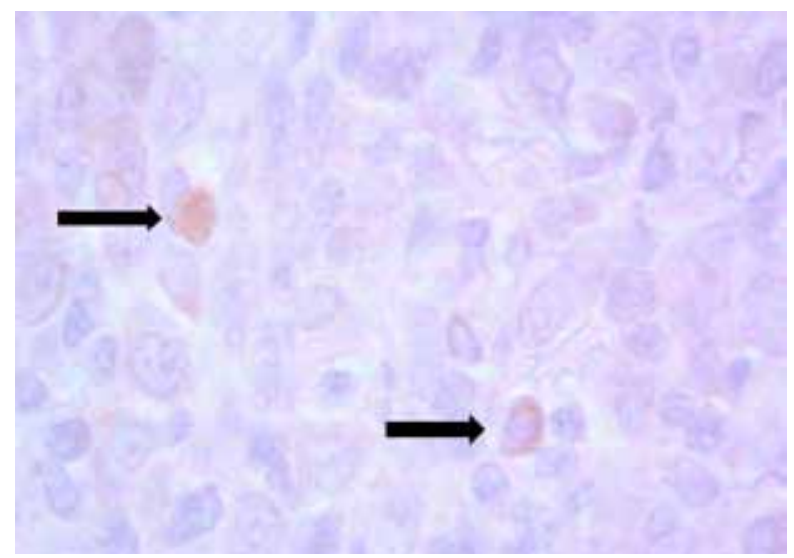

Figura 4. Misma biopsia de la Figura 3, resaltando mediante inmunomarcación la presencia de linfocitos Th1. Las flechas indican dos células inmunomarcadas (IHQ 100X). tras con baja carga parasitaria superaban las 8 células por campo de mayor aumento.

\section{DISCUSIÓN}

Cuando las células dendríticas toman contacto con antígenos de LV, sufren un proceso de maduración por lo cual aumentan en su superficie la expresión del complejo mayor de histocompatibilidad. Estas células dendríticas infectadas sintetizan IL-12, que conduce a generar un perfil de tipo Th1 ${ }^{24}$. Las respuestas colaboradoras de Th1 son efectivas para eliminar patógenos que se hallan dentro de las células, mientras que las de tipo Th2 son eficaces para la eliminación de microorganismos extracelulares y parásitos ${ }^{1}$.

En la leishmaniosis, la expansión de células Th1 se asocia a inmunidad protectora y la de Th2 a progresión de la enfermedad y persistencia ${ }^{2,3,13}$. Si bien hay estudios que indican que la LV se caracteriza por aparición de síntomas clínicos dependiendo de la carga parasitaria en diferentes órganos ${ }^{19,20}$, en este estudio la presencia o ausencia de signos clínicos en muchos casos no estuvo relacionada a la cantidad de parásitos encontrados en las biopsias de piel.

Se han encontrado animales sin sintomatología y con elevada cantidad de parásitos presentes en la biopsia, así como animales con baja carga parasitaria y sintomatología variable. Por otra parte, se observa que la alta carga parasitaria se relaciona con inmunosupresión mediada por linfocitos Thl, mientras que en los animales con alta respuesta inmunológica, la carga parasitaria está disminuida.

Los datos obtenidos coinciden con autores que manifiestan que el control de la LV depende de una exitosa respuesta inmune mediada por células ${ }^{15}$, en la cual el IFN- $\gamma$ producido por las células Th1 y NK estimuladas por IL-12, lleva a la acción microbicida mediada por óxido nítrico.

\section{REFERENCIAS}

1. Abbas AK, Murphy KM, Sher A. 1996. Functional diversity of helper T lymphocytes. Nature 383: 787-793.

2. Akuffo H, Maasho K, Britton S. 1996. Immunity to human leishmaniasis. Parasitology of the 21st Century, $C A B$ International 23-33.

3. Alexander J, Bryson K. 2005. T helper (h)1/Th2 and Leishmania: paradox rather than paradigm. Immunol Lett 99: 17-23.

4. Antoine JC, Prina E, Courret N, Lang T. 2004. Leishmania spp.: on the interactions they establish with antigenpresenting cells of their mammalian hosts. Adv Parasitol 58: 1-68.

5. Bhattacharya P, Ali N. 2013. Involvement and interactions of different immune cells and their cytokines in human visceral leishmaniasis. Rev Soc Bras Med Trop 46: 128-134.

6. Banchereau $\mathbf{J}$ et al. 2000. Immunobiology of dendritic cells. Ann Rev Immunol 18: 767-811. 
7. Bogdan C, Rollinghoff M, Diefenbach A. 2000. The role of nitric oxide in innate immunity. Immunol Rev 173: 17-26.

8. Brandonisio $\mathbf{O}$ et al. 2002. Macrophage chemotactic protein-1 macrophage inflammatory protein-l alpha induce nitric oxide release and enhance parasite killing in Leishmania infantum infected human macrophages. Clin Exp Med 2: 125-129.

9. Carvalho EM, Bacellar O, Barral A, Badaro R, Johnson WD. 1989. Antigen-specific immuno suppression in visceral leishmaniasis is cell mediated. $J$ Clin Invest 83: 860-864.

10. Costa DL et al. 2013. Serum cytokines associated with severity and complications of kala-azar. Pathog Glob Health 107: 78-87.

11. Dujardin JC et al. 2008. Spread of vector-borne diseases and neglect of leishmaniasis, Europe Emerg Infect Dis 14: 1013-1018.

12. Lima GM et al. 1998. The role of polymorphonuclear leukocytes in the resistance to cutaneous leishmaniasis. Immunol Lett 64: 145-151.

13. Lockesley RM, Louis JA. 1992. Immunology of leishmaniasis. Curr Opin Immunol 4: 413-418.

14. Manna PP, Chakrabarti G, Bandyopadhyay S. 2010. Innate immune defense in visceral leishmaniasis: cytokine mediated protective role by allogeneic effector cell. Vaccine 28: 803-810.

15. Murray HW. 1997. Endogenous interleukin-12 regulates acquired resistance in experimental visceral leishmaniasis. J Infect Dis 175: 1477-1479.
16. Murray HW, Berman JD, Davies CR, Saravia NG. 2005. Advances in leishmaniasis. Lancet 366: 1561-1577.

17. Nylen S, Sacks D. 2007. Interleukin-10 and the pathogenesis of human visceral leishmaniasis. Trends Immunol 28 : 378-384.

18. Peruhype MV et al. 2005. Immune response in human visceral leishmaniasis: analysis of the correlation between innate immunity cytokine profile and disease outcome. Scand J Immunol 62: 487-495.

19. Reis AB et al. 2006. Parasite density and impaired biochemical / hematological status are associated with severe clinical aspects of canine visceral leishmaniasis. Res Vet Sci 81: 68-75.

20. Reis AB et al. 2009. Systemic and compartmentalized immune response in canine visceral leishmaniasis. Vet Immunol Immunopathol 128: 87-95.

21. Santos GM et al. 2002. Cytokine expression during the outcome of canine experimental infection by Leishmania infantum. Vet Immunol Immunopathol 88: 21-30.

22. Vangisbergen KP, Geijtenbeek TB, Vankooyk Y. 2005. Close encounters of neutrophils and DCs. Trends Immunol 26: 626-631.

23. Vangriensven J, Diro E. 2012 Visceral leishmaniasis. 2012. Infect Dis Clin North Am 26: 309-322.

24. Vonstebut E, Udey MC. 2004. Requirements for Th1dependent immunity against infection with Leishmania major. Microbes Infect 6: 1102-1109. 